\title{
Preface
}

\section{Advances in Cardiac Mapping: Part 1}

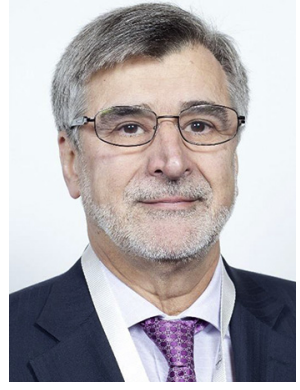

Mohammad Shenasa, MD, FHRS

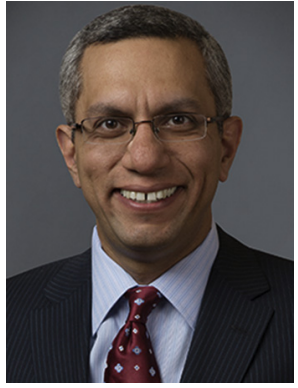

Amin Al-Ahmad, MD, FHRS

Editors

Cardiac mapping has come a long way, and it has been an integral part of interventional cardiac electrophysiology. Cardiac mapping started with direct single-analogue point-by-point registration of cardiac electrical activity to its utmost complex online multimodality mapping and imaging. Technological advances in cardiac mapping and ablation allowed rhythmologists and interventional electrophysiologists to better understand the mechanisms and management of arrhythmias. Despite the unprecedented technological advances in the diagnosis and management of cardiac arrhythmias, such as atrial fibrillation, epicardial ventricular tachycardias, and arrhythmias in congenital heart disease, challenges remain ahead.

The future lies in the noninvasive mapping and imaging in the diagnosis of cardiac arrhythmias, such as electrocardiographic imaging, in even a single cardiac beat to the noninvasive ablation techniques, such as noninvasive cardiac radiation and zero to near-zero fluoroscopy, to avoid radiation exposure.

We are pleased that the consulting editors of Cardiac Electrophysiology Clinics. Ranjan K. Thakur, MD, and Andrea Natale, MD, invited us to serve as editors for this important topic. In addition, we are delighted that a group of pioneers in the field of cardiac mapping and ablation has unanimously accepted our invitation to contribute their state-of-the-art articles for this and the next issue of Cardiac Electrophysiology Clinics, both on advances in cardiac mapping.
We initially planned to have a single issue dedicated to this topic. However, due to important topics that needed to be covered, we were obliged to include a 2-issue comprehensive review. Part 1 covers the basic concepts, including cardiac embryology and anatomy relevant to cardiac mapping and ablation, followed by novel mapping and imaging techniques, such as cardiac computed tomography and $\mathrm{MRI}$, and catheter and energy sources. Part 2 discusses mapping and ablation of particular arrhythmias in specific substrates, such as atrial fibrillation, ventricular tachycardia, and fibrillation.

We are confident that these 2 issues on advances in cardiac mapping will be useful to clinical cardiac electrophysiologists, fellow trainees, attending, and allied professionals who participate in the management of patients with complex arrhythmias, and we hope that it improves patients' quality of life and survival.

Mohammad Shenasa, MD, FHRS Department of Cardiovascular Services O'Connor Hospital Heart and Rhythm Medical Group 105 North Bascome Avenue San Jose, CA 95128, USA

Amin Al-Ahmad, MD, FHRS Texas Cardiac Arrhythmia Institute St David's Medical Center Austin, TX 78705, USA

E-mail addresses:

mohammad.shenasa@gmail.com (M. Shenasa) aalahmadmd@gmail.com (A. Al-Ahmad) 